\title{
Can Proximal Junctional Kyphosis After Surgery for Adult Spinal Deformity Be Predicted by Preoperative Dynamic Sagittal Alignment Change With 3D Gait Analysis? A Case-control Study
}

\section{Tomoyuki Asada ( $\nabla$ kjjk991@tsukuba-seikei.jp )}

Department of Orthopaedic Surgery, Faculty of Medicine, University of Tsukuba

Kousei Miura

Department of Orthopaedic Surgery, Faculty of Medicine, University of Tsukuba

Masao Koda

Department of Orthopaedic Surgery, Faculty of Medicine, University of Tsukuba Hideki Kadone

Center for Innovative Medicine and Engineering, University of Tsukuba Hospital

\section{Toru Funayama}

Department of Orthopaedic Surgery, Faculty of Medicine, University of Tsukuba Hiroshi Takahashi

Department of Orthopaedic Surgery, Faculty of Medicine, University of Tsukuba Hiroshi Noguchi

Department of Orthopaedic Surgery, Faculty of Medicine, University of Tsukuba

\section{Yosuke Shibao}

Department of Orthopaedic Surgery, Faculty of Medicine, University of Tsukuba

\section{Kosuke Sato}

Department of Orthopaedic Surgery, Faculty of Medicine, University of Tsukuba

\section{Fumihiko Eto}

Department of Orthopaedic Surgery, Faculty of Medicine, University of Tsukuba

\section{Mamoru Kono}

Department of Orthopaedic Surgery, Faculty of Medicine, University of Tsukuba Kentaro Mataki

Department of Orthopaedic Surgery, Faculty of Medicine, University of Tsukuba Masashi Yamazaki

Department of Orthopaedic Surgery, Faculty of Medicine, University of Tsukuba

\section{Research Article}


Keywords: PJK, 3D gait analysis, adult spinal deformity, degenerative lumbar kyphoscoliosis, sagittal alignment, dynamic alignment

Posted Date: November 16th, 2021

DOI: https://doi.org/10.21203/rs.3.rs-1025712/v1

License: (1) This work is licensed under a Creative Commons Attribution 4.0 International License. Read Full License 


\section{Abstract}

\section{Purpose}

To investigate whether preoperative dynamic spinal alignment that worsened during gait as detected by three-dimensional (3D) gait analysis can be a predictive factor for proximal junctional kyphosis (PJK) after corrective surgery for adult spinal deformity (ASD) with a minimum 1-year follow-up.

Method

We included 27 patients with ASD who underwent 3D gait analysis before corrective surgery and had $>1$ year follow-up. Dynamic spinal alignment parameters were obtained using a Nexus motion capture system (Vicon, Oxford, UK) with reflective markers. Spinal alignment was assessed in each region and pelvic alignment was assessed with surface markers. Patients were asked to walk for as long as possible around an oval walkway. We obtained the averaged dynamic parameters in the final lap and compared them between patients with PJK(+) and those with PJK(-).

Results

PJK occurred in 7 patients (26\%). Preoperative angle between the thoracic spine and the pelvis was larger in patients with $\mathrm{PJK}(+)$ than in those with $\mathrm{PJK}(-)(32.3 \pm 8.1$ vs $18.7 \pm 13.5, P=0.020)$ with sufficient sample size. Multiple logistic regression analysis identified the angle between the thoracic spine and pelvis as an independent risk for PJK.

\section{Conclusion}

Preoperative thoracic kyphosis exacerbated by gait as determined in 3D gait analysis is a preoperative independent risk factor of PJK in patients undergoing ASD corrective surgery.

\section{Introduction}

Corrective surgery for adult spinal deformity (ASD) is a principal means to improve the quality of life by restoring whole spinal alignment ${ }^{1}$. Proximal junctional kyphosis (PJK) is generally defined as a proximal junctional sagittal Cobb angle $>10^{\circ}$ between the lower endplate of the uppermost instrumented vertebra and the upper endplate of two supra-adjacent vertebral bodies $\geq 10^{\circ}$ greater than the preoperative measurement ${ }^{2}$, and is a representative complication of corrective surgery for ASD. It occasionally requires extended instrumentation surgery for instrument failure, sagittal malalignment, and neurological compromise. The incidence of PJK was previously reported as $20-40 \%{ }^{2-7}$, representing a substantial burden for patients. As risk factors for PJK, patient- and surgically-related factors such as older patients, low bone mineral density (BMD), severe spinal malalignment, and longer correction surgery were previously reported ${ }^{7}$. A meta-analysis investigated other risk factors, and $>20$ was indicated, but they are difficult to apply to clinical practice ${ }^{8}$ because the severe deformity itself is a characteristic of ASD. Thus, 
prevention of PJK remains to be established despite numerous efforts to overcome this complication ${ }^{9-}$ 12.

Although worsening spinal alignment during gait was reported in patients with ASD ${ }^{13}$, it can barely be detected using conventional standing X-ray images. Three-dimensional (3D) gait analysis can provide quantitative assessment of spinopelvic alignment change and failure of compensatory mechanisms

during gait ${ }^{13-16}$. Among patients with spinal malalignment that gait exacerbates, static standing X-ray image assessment can result in underestimating severe spinal deformity. Even if these patients are at a high risk of PJK, they cannot be assessed adequately by conventional assessment before surgery. Thus, we hypothesized that PJK would be affected by preoperative dynamic spinopelvic alignment change during gait. In this study, we sought to investigate whether preoperative dynamic spinal alignment aggravated by gait as determined by 3D gait analysis can be a risk factor for PJK.

\section{Results}

\section{Patient inclusion and demographic data}

We first included 36 patients with ASD who underwent gait analysis and corrective surgery. Nine patients met the exclusion criteria, and therefore 27 patients were ultimately included in this analysis (Fig. 1).

The cohort included 7 male and 20 female patients. PJK occurred in 7 (26\%, 1 male, and 6 female). There was no significant difference in age, height, body weight, or BMD. Both sagittal and radiographic parameters, including C7SVA, TK, LL, PI-LL, were not significantly different preoperatively (Table 1). 
Table 1

Patient demographic

\begin{tabular}{|c|c|c|c|c|c|c|}
\hline \multirow{2}{*}{$\mathrm{n}$} & \multirow[t]{2}{*}{ Parameter } & \multicolumn{2}{|l|}{ PJK(+) } & \multirow{2}{*}{$\begin{array}{l}\text { PJK(-) } \\
20\end{array}$} & \multicolumn{2}{|r|}{$P$} \\
\hline & & 7 & & & & \\
\hline \multicolumn{2}{|c|}{ Sex (male, female) } & 1 & 6 & 4 & 16 & \\
\hline \multicolumn{2}{|c|}{ Age (years) } & 67.5 & \pm 6.24 & 68.6 & \pm 10.2 & 0.969 \\
\hline \multicolumn{2}{|c|}{ Height $(\mathrm{cm})$} & 146.7 & \pm 7.8 & 151.1 & \pm 35.3 & 0.340 \\
\hline \multicolumn{2}{|c|}{ Body weight (kg) } & 52.9 & \pm 12.3 & 49.6 & \pm 14.1 & 0.668 \\
\hline \multicolumn{2}{|c|}{$\mathrm{BMD}\left(\mathrm{g} / \mathrm{cm}^{2}\right)$} & 0.63 & \pm 0.1 & 0.60 & \pm 0.2 & 0.669 \\
\hline \multicolumn{2}{|c|}{ YAM (\%) } & 77.3 & \pm 8.6 & 73.6 & \pm 25.9 & 0.635 \\
\hline \multirow[t]{9}{*}{ PreOp } & C7SVA (mm) & 115.9 & \pm 51.9 & 112.7 & \pm 58.1 & 0.900 \\
\hline & $\operatorname{TK}\left({ }^{\circ}\right)$ & 20.5 & \pm 11.8 & 16.3 & \pm 15.6 & 0.524 \\
\hline & $\operatorname{LL}\left({ }^{\circ}\right)$ & 6.3 & \pm 16.8 & 11.7 & \pm 20.3 & 0.703 \\
\hline & $\mathrm{PT}\left({ }^{\circ}\right)$ & 36.7 & \pm 9.4 & 31.6 & \pm 14.8 & 0.436 \\
\hline & $\mathrm{PI}\left({ }^{\circ}\right)$ & 52.3 & \pm 12.8 & 49.6 & \pm 15.4 & 0.501 \\
\hline & $\operatorname{TPA}\left({ }^{\circ}\right)$ & 39.7 & \pm 11.4 & 35.5 & \pm 16.7 & 0.625 \\
\hline & $\operatorname{PILL}\left({ }^{\circ}\right)$ & 46.0 & \pm 19.5 & 37.8 & \pm 23.2 & 0.463 \\
\hline & C7CSVL (mm) & 3.6 & \pm 31.3 & 4.6 & \pm 28.9 & 0.742 \\
\hline & $\operatorname{Cobb}\left(^{\circ}\right)$ & 37.4 & \pm 21.7 & 24.1 & \pm 15.5 & 0.102 \\
\hline \multicolumn{7}{|c|}{$\begin{array}{l}\text { Mean } \pm \text { standard deviation; } n \text {, number of patients; BMD, bone mineral density; YAM, young adult } \\
\text { mean; C7SVA, C7 plumb line to sagittal vertical axis distance; TK, thoracic kyphosis; LL, lumbar } \\
\text { lordosis; PT, pelvic tilt; PI, pelvic incidence; TPA, T1 pelvic angle; PI-LL, PI minus LL; C7CSVL, C7 } \\
\text { plumb line to central sacral vertical line distance; PreOp, preoperative parameter }\end{array}$} \\
\hline \multicolumn{7}{|c|}{$\begin{array}{l}\text { Vertebral body corpectomy was performed for } 2 \text { patients with PJK(+) and } 2 \text { with PJK( }(-) \text {. In the } \\
\text { postoperative assessment, in standing X-ray images, C7SVA, TK and LL were significantly greater, and } \\
\text { PI-LL was significantly smaller in PJK (+) (TK, } 49.31 \pm 12.23 \text { vs } 26.29 \pm 15.3, P=0.002 ; \mathrm{LL}, 54.03 \pm \\
10.79 \text { vs } 34.50 \pm 17.68, P=0.012 \text {; PI-LL, }-8.97 \pm 12.25 \text { vs } 9.42 \pm 20.12, P=0.033) \text { (Table } 2) \text {. }\end{array}$} \\
\hline
\end{tabular}


Table 2

Postoperative spinal parameters

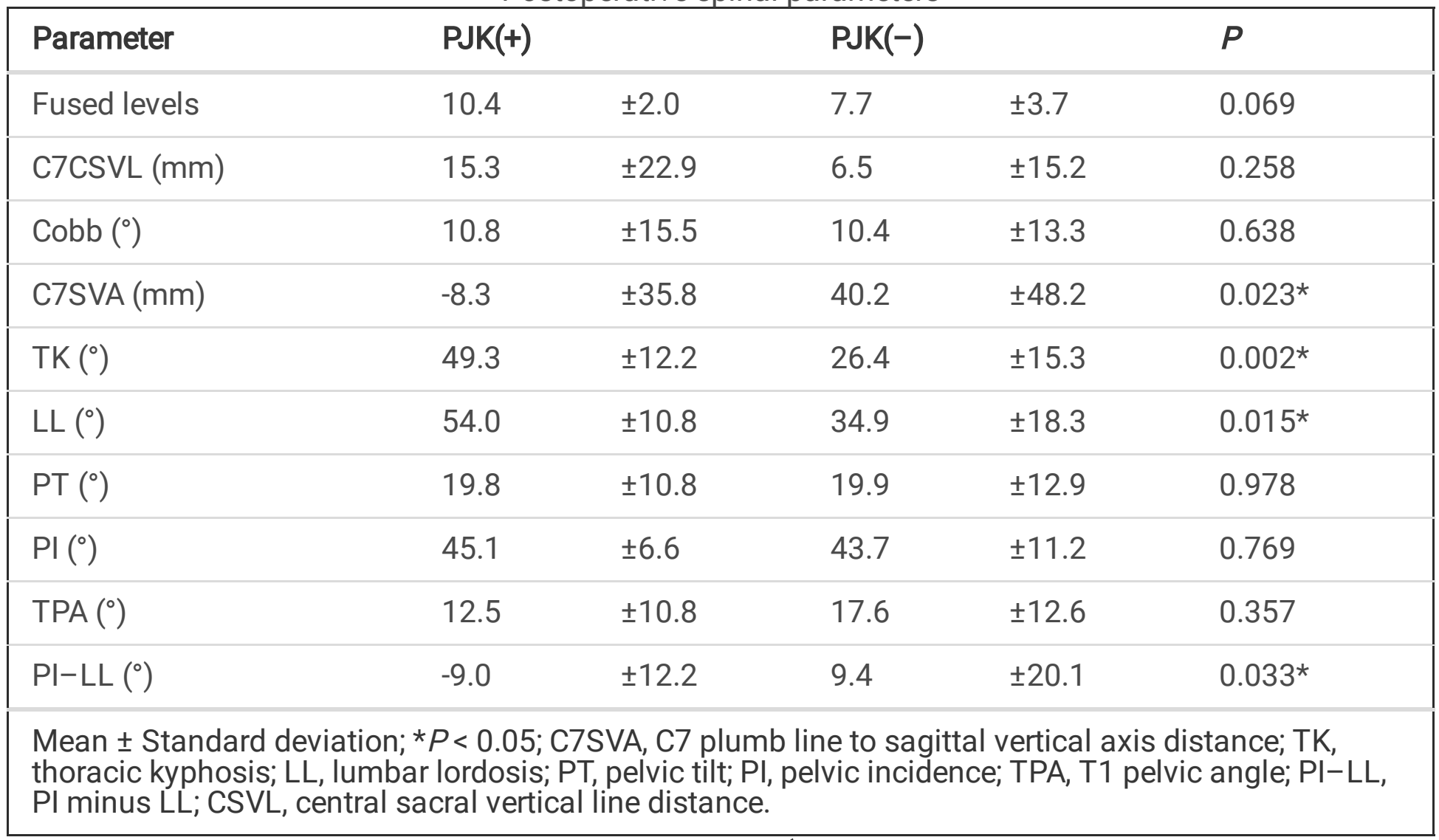

\section{Three-dimensional gait analysis (dynamic spinal parameters)}

In the preoperative 3D gait analysis, thoracic-pelvic spinal angle (T-PSA) in patients with PJK(+) was significantly larger than that in those with PJK(-) (32.21 \pm 8.06 vs $18.72 \pm 13.54, P=0.020)$. There was no significant difference in any parameter except T-PSA (Table 3). 
Table 3

Dynamic sagittal parameters between each group

\begin{tabular}{|c|c|c|c|c|c|}
\hline Parameter & PJK(+) & & PJK(-) & & $P$ \\
\hline T-SVA (mm) & 158.4 & \pm 36.8 & 118.2 & \pm 46.6 & 0.050 \\
\hline L-SVA (mm) & 15.0 & \pm 21.3 & 27.4 & \pm 31.8 & 0.351 \\
\hline S-SVA $(\mathrm{mm})$ & 194.8 & \pm 56.2 & 165.1 & \pm 74.5 & 0.347 \\
\hline T-SA $\left(^{\circ}\right)$ & 33.5 & \pm 9.2 & 25.1 & \pm 12.1 & 0.107 \\
\hline $\operatorname{L-SA}\left({ }^{\circ}\right)$ & 4.9 & \pm 8.1 & 10.0 & \pm 11.8 & 0.300 \\
\hline S-SA $\left(^{\circ}\right)$ & 25.0 & \pm 7.2 & 20.4 & \pm 9.9 & 0.278 \\
\hline P-SA $\left(^{\circ}\right)$ & 92.4 & \pm 4.4 & 90.2 & \pm 22.3 & 0.802 \\
\hline T-P SA $\left(^{\circ}\right)$ & 32.3 & \pm 8.1 & 18.7 & \pm 13.5 & $0.020 *$ \\
\hline L-P SA $\left({ }^{\circ}\right)$ & -1.9 & \pm 14.1 & 5.1 & \pm 11.2 & 0.193 \\
\hline S-P SA $\left({ }^{\circ}\right)$ & 22.5 & \pm 7.2 & 14.5 & \pm 12.1 & 0.116 \\
\hline \multicolumn{6}{|c|}{ Mean \pm Standard deviation; $* P<0.05$} \\
\hline \multicolumn{6}{|c|}{ SVA, sagittal vertical axis; SA, sagittal angle; P SA, pelvic sagittal angle } \\
\hline \multicolumn{6}{|c|}{$\begin{array}{l}\text { Other sagittal parameters (T-SVA, L-SVA. S-SVA, T-SA, L-SA, S-SA, L-PSA, S-PSA) were not significantly } \\
\text { different. There was no difference in coronal alignment (Table 4). }\end{array}$} \\
\hline
\end{tabular}


Table 4

Dynamic coronal parameters between each group

\begin{tabular}{|c|c|c|c|c|c|}
\hline Parameter & PJK(+) & & PJK(- & & $P$ \\
\hline T-CVA (mm) & 10.8 & \pm 31.7 & 8.2 & \pm 19.9 & 0.802 \\
\hline L-CVA (mm) & 1.4 & \pm 13.8 & -3.0 & \pm 19.5 & 0.589 \\
\hline S-CVA (mm) & 11.2 & \pm 31.4 & 7.7 & \pm 34.6 & 0.819 \\
\hline $\mathrm{T}-\mathrm{CA}\left({ }^{\circ}\right)$ & 3.4 & \pm 8.4 & 2.2 & \pm 5.1 & 0.655 \\
\hline $\mathrm{L}-\mathrm{CA}\left({ }^{\circ}\right)$ & 0.6 & \pm 5.5 & 0.4 & \pm 9.5 & 0.960 \\
\hline S-CA $\left({ }^{\circ}\right)$ & 1.7 & \pm 4.6 & 1.4 & \pm 5 & 0.909 \\
\hline $\mathrm{P}-\mathrm{CA}\left({ }^{\circ}\right)$ & 88.5 & \pm 7.5 & 86.1 & \pm 20.3 & 0.764 \\
\hline $\mathrm{T}-\mathrm{P}$ CA $\left({ }^{\circ}\right)$ & 5.1 & \pm 12.2 & -0.2 & \pm 11.1 & 0.305 \\
\hline L-P CA $\left({ }^{\circ}\right)$ & -4.2 & \pm 11.8 & -0.4 & \pm 10.7 & 0.435 \\
\hline S-P CA $\left({ }^{\circ}\right)$ & 3.1 & \pm 9.2 & -0.7 & \pm 8.2 & 0.326 \\
\hline \multicolumn{6}{|c|}{ Mean \pm standard deviation } \\
\hline \multicolumn{6}{|c|}{ CVA, coronal vertical axis; CA, coronal angle; P CA, pelvic coronal angle } \\
\hline \multicolumn{6}{|c|}{$\begin{array}{l}\text { The potential variables (T-PSA and L-PSA) were included in a multiple logistic regression model. This } \\
\text { model found that T-PSA was an independent preoperative factor having a significant association with } \\
\text { PJK (OR, } 1.23 ; 95 \% \mathrm{Cl}, 1.031-1.477 ; P=0.0005) \text {. A Hosmer-Lemeshow test result found the model to } \\
\text { be a good fit }(P=0.858) \text {. }\end{array}$} \\
\hline \multicolumn{6}{|c|}{ Post hoc power analysis of Student's t-test } \\
\hline \multicolumn{6}{|c|}{$\begin{array}{l}\text { We performed a post hoc power analysis for unpaired two-group comparisons with significant } \\
\text { difference. The effect size was calculated from the mean and standard deviation of T-PSA of each } \\
\text { group and determined as } 1.48 \text {. This effect size and sample size of this analysis indicated that the } \\
\text { power ( } 1-\beta \text { error probability) was } 0.90 \text {, indicating adequate sample size. }\end{array}$} \\
\hline
\end{tabular}

\section{Representative case}

A 64-year-old woman with adult spinal deformity and 3D gait analysis before surgery underwent corrective surgery for her main complaint of low back pain. The sagittal parameters on preoperative standing X-ray imaging were as follows: C7SVA, $54.2 \mathrm{~mm}$; TK, 33.1 ${ }^{\circ}$; LL, 41.7 $; \mathrm{PT}, 20.7^{\circ} ; \mathrm{PI}, 50.2^{\circ}$; TPA, $19.8^{\circ}$ (Fig. 2a). PJK was detected in postoperative X-ray imaging at 6 months. At the postoperative year 1 follow-up, PJA was $31.2^{\circ}$ and progression of PJA was $11.2^{\circ}$ (Fig. $2 \mathrm{~b}$ and $2 \mathrm{c}$ ).

Figure 3 indicates the posture during gait analysis. Before starting to walk, the patient seemed relatively balanced in a standing upright position before gait analysis (Fig. 3a). Starting the walk, the thoracic part began to lean forward in the first lap (Fig. 3b). In the final lap soon before quitting the trial, the tilt of the thoracic spine leaned further forward (Fig. 3c). The superimposed image of posture during gait showed a 
change of the thoracic spine tilt between the first (T-P SA $17.91^{\circ}$ ) and the final lap (T-P SA $26.94^{\circ}$ ) (Fig. 3d). This patient complained of implant eminence and pain in the proximal junctional area but refused reoperation.

\section{Discussion}

The present study investigated the association of preoperative dynamic spinal malalignment exacerbated by gait with postoperative PJK incidence. Preoperative T-PSA was larger in patients with PJK(+) than it was in those with PJK(-) (32.84 \pm 7.76 vs $18.53 \pm 13.40, P=0.014)$ with sufficient sample size. The multiple logistic regression analysis revealed preoperative T-PSA was an independent factor significantly associated with PJK (odds ratio, 1.23; $95 \% \mathrm{Cl}, 1.031-1.477 ; P=0.0005$ ). Preoperative demographic data of both groups indicated no significant difference in age, osteoporosis, or sagittal alignment in standing X-ray images. Fused level and TK showed a difference in the postoperative assessment.

T-PSA is the thoracic sagittal angle between the C7-T12 connecting line and ASIS-PSIS connecting line. The larger T-PSA in the final lap suggested the thoracic spine leaned forward from the standing position to the final lap. The forward lean of the thoracic spine could be interpreted as a thoracic or thoracolumbar junctional kyphosis. T-SA is the angle between the gravity line and the thoracic spine and P-SA is the angle between the pelvis and the horizontal line, both of which could be compensated by pelvic retroversion and knee-flexion respectively. SVA is one of the most widely known parameters indicating the severity of deformity and is exacerbated while walking ${ }^{17}$, but could also be directly influenced by compensation from pelvic retroversion and knee flexion. By contrast, T-PSA, which is defined as the angle between the thoracic spine and the pelvis, could subtract the effect of pelvic retroversion and was independent of knee flexion. Therefore, T-SA, P-SA, and spinal SVA were not different between the two groups, whereas T-PSA was significantly different between PJK(+) and PJK(-) groups. The compensation mechanism of the pelvis and lower leg worked equally to maintain spinal balance in both groups even soon before walking ended, while the patients in the PJK(+) group could not compensate for thoracic kyphosis while walking. A possible mechanism for decompensated spinal alignment during gait is considered to be the weakness of the paraspinal muscles of the relevant area. A relationship between PJK and lower muscularity at thoracolumbar area has been reported ${ }^{18,19}$. Together with the results of the present study, it is possible that the lower muscularity of certain spinal muscles can lead to disruption of compensatory mechanisms that maintain alignment in the thoracic spine while walking, and that this disruption was elicited by walking. Therefore, we may have underestimated the risk of PJK in patients with mild deformity as seen in standing X-ray images. Further investigation of the relationship between the muscularity and occurrence of PJK is warranted because of the lack of present evidence.

In the postoperative X-ray image assessment, TK and LL were significantly larger and PI-LL was significantly lower in patients with $\mathrm{PJK}(+)$. $\mathrm{PI}-\mathrm{LL}<0$ has been reported as a risk factor for PJK ${ }^{20}$. Excessive postoperative LL for PI results in a PI-LL mismatch as well as inadequate correction. Patients with an excessively flexible thoracic spine preoperatively are more likely to have reciprocal change in unfused thoracic spine leading to development of PJK. Age adjusted appropriate alignment is suggested 
as a goal for ideal alignment to prevent PJK in the elderly ${ }^{21}$, while another study emphasized the importance of strict correction to achieve better ODI outcomes especially in elderly Japanese ${ }^{22}$. These studies indicated that we need to consider the optimal alignment on a patient-by-patient basis. Patients with a tendency for the thoracic spine to tilt forward during walking may have an over-reciprocal change after corrective surgery for ASD, but to our knowledge, no previous study has investigated the relationship between spinal alignment during gait and spinal change after surgery. Therefore, adequate alignment in patients with a large TPSA after walking should be investigated in future study.

A strong point of the present study is the unique analysis method and novelty. To our knowledge, there is only one previous report about the relationship between gait analysis and operative results. Ham et al. reported that anterior pelvic tilt angle is an independent risk factor for PJK by preoperative motion analysis ${ }^{23}$. The calculation of anterior pelvic tilt used was the same as P-SA in the present study, which was not significantly different here. Anterior pelvic tilt was calculated using average variables over the entire gait cycle. A possible cause of this discrepancy might be the measurement method. Our analysis required that patients walk for as long as possible to establish their fatigue status, and we averaged the parameters from only the final lap. The advantages of each study are unclear, but spinal alignment was different when the first lap and the final lap were compared ${ }^{17}$. The patient fatigue status and spinal alignment at that time should be interpreted carefully.

Some limitations should be addressed. First, we did not investigate the patient-reported outcome of QOL. PJK was reported as having no effect on a patient's QOL, but subsequent studies have gradually clarified its relationship with QOL. It is still unclear whether all patients with a large T-PSA should undergo additional surgery to prevent PJK. Second are differences in the number of fused levels. The results of the patient demographic analysis indicated patients included in this study were relatively uniform especially before the surgery. However, fused level and postoperative TK was significantly different in each group. Surgeons choose a higher UIV leading to a long fused level in patients with severe deformity or higher TK in standing radiographs. The deformity of each patient should be assessed independently. Thus, it was difficult to make an identical comparison of fused level. Third is the possibility of selection bias. This study included the patients with corrective surgery and preoperative gait analysis. It is ideal that all patients underwent gait analysis before corrective surgery, but the indication of gait analysis was determined by the patient and examination room schedule before surgery. Therefore, an ideal analysis could not always be accomplished. Finally, the accuracy of the reflective marker on the skin should be considered. Soft tissue thickness is difficult to overcome, especially on the lumbar spine ${ }^{24}$. However, the 3D gait analysis system in the present study has included the influence of soft tissue on the accuracy of the marker placement. Moreover, the influence of the lumbar lordosis was minimized in the analyzed population of the present study because most patients had reduced lumbar lordosis due to ASD.

In conclusion, the present study indicated that thoracic kyphosis exacerbated by gait as determined by 3D gait analysis is a preoperative independent risk factor for PJK in patients with ASD undergoing corrective surgery. By contrast, lumbar spinal change was not significantly different between patients with PJK(+) or 
PJK(-). To assess risk, dynamic thoracic kyphosis change should be assessed carefully, rather than lumbar spine alignment alone.

\section{Methods}

\section{Study design and participants data}

We conducted an observational case-control study within a cohort of patients who underwent spine surgery. We included patients with ASD who underwent corrective surgery over 3 spinal levels and gait analysis at our university hospital between December 2015 and March 2020. Radiographic inclusion criteria were as follows: able to stand without any support during X-ray imaging; pelvic incidence minus lumbar lordosis $(\mathrm{PI}-\mathrm{LL})>10^{\circ}$; sagittal vertical axis $(\mathrm{SVA})>4 \mathrm{~cm}$; and pelvic tilt $(\mathrm{PT})>20^{\circ}$, as spinal parameters related to sagittal malalignment according to the SRS-Schwab ASD classification. ${ }^{25}$ Exclusion criteria were as follows: (1) proximal junctional failure after another spinal surgery; (2) unable to stand alone during X-ray imaging because of pain or weakness in lower extremities; and (3) <1-year follow-up after surgery.

Demographic data included sex, height, body weight, bone mineral density (BMD) by dual-energy X-ray absorptiometry (DEXA) and fused spinal level. ASD is often complicated by vertebral fractures, and the lumbar DEXA is likely to be higher than the actual bone density, which is reported to deviate significantly from the hip DEXA ${ }^{26}$. In addition, lumbar DEXA does not correlate well with vertebral body failure load ${ }^{27}$. Based on these reports, we used BMD of the femur because discrepancies due to osteosclerosis after vertebral body fracture were expected. Radiographical assessments are listed in the Radiographic assessment section.

The present study was conducted within an appropriate ethical framework, and in accordance with the Declaration of Helsinki and its contemporary amendments. Approval from the ethics committee of our local ethics committee (Tsukuba Clinical Research and Development Organization) (approval number: H26-144) was obtained for this study design. Written informed consent was obtained from all patients included in this study.

\section{Radiographic assessment}

We assessed the whole spine parameters digitally before surgery as a static evaluation. Spinal parameters included sagittal vertical axis (SVA); thoracic kyphosis (TK); lumbar lordosis (LL); pelvic tilt (PT); pelvic incidence (PI); T1 pelvic angle (TPA); coronal Cobb angle of the thoracolumbar and lumbar scoliosis (Cobb); and coronal balance (C7-CSVL, the distance between a C7 plumb line and the center sacral vertical line). PI-LL was calculated from values obtained. All patients were asked to stand usually and look straight ahead in the radiographic exam. PJK was defined as a proximal junctional angle (PJA) $>10^{\circ}$ soon after operation and $>10^{\circ}$ progression of PJA ${ }^{2}$. PJA and postoperative spinal parameters were assessed at 1 year after surgery. Using this definition of PJK, patients were classified into PJK(+) and PJK(-) groups. There were no patients who required additional surgery before 1 year postoperatively. 


\section{Surgical procedure}

All surgeries in this analysis were performed or supervised by the senior authors who are experienced board-certified spinal surgeons. For the anterior segment of the lumbar spine, extreme lateral interbody fusion (XLIF®, Nuvasive, SanDiego, CA, USA) with a mini-open technique was conducted to achieve correction. Total facetectomy as grade 2 osteotomy were performed in the lumbar spine when needed to achieve sufficient correction ${ }^{28}$. Higher grade osteotomy than grade 2 were not performed in the present series. Anterior vertebral body corpectomy with an expandable cage was performed in patients with deformity caused by vertebral collapse. Subsequently, posterior decompression and correction with an open approach and pedicle screw system were performed. Total facetectomy was performed when needed to achieve sufficient correction. Posterior column osteotomy or pedicle subtraction osteotomy were not performed in the present series. We did not add hooks, sublaminar taping, cement augmentation, or other preventive surgical techniques to avoid PJK. The chief surgeon decided the upper instrumented vertebra (UIV) and the lower instrumented vertebra (LIV) depending on each case, and the decision was approved as our consensus.

\section{Gait analysis and dynamic spinal parameter}

3D gait analysis was conducted using a Nexus motion capture system (Vicon, Oxford, UK) comprising 16 cameras and 38 reflective makers variously attached on the head, spinal spinous processes, pelvis, and upper and lower limbs of the patients (Fig. 4).

Trials consisted of walking for as long as the patients could around an oval walkway in a laboratory room. Patients could stop whenever they felt too fatigued to walk more. The walkway was comprised of two parallel $10 \mathrm{~m}$ straight paths and two semicircular paths of approximately $1 \mathrm{~m}$ radius. Table 5 lists the spinal markers and dynamic spinal parameters obtained from gait analysis. 
Table 5

Dynamic spinal alignment parameters (mean \pm SD)

\begin{tabular}{|c|c|c|c|c|}
\hline Parameter & Definition & Mark & & Unit \\
\hline T-SVA & Thoracic sagittal distance between the reflective markers & $\mathrm{C} 7$ & T12 & $\mathrm{mm}$ \\
\hline T-CVA & Thoracic coronal distance between the reflective markers & $\mathrm{C7}$ & $\mathrm{T} 12$ & $\mathrm{~mm}$ \\
\hline L-SVA & Lumbar sagittal distance between the reflective markers & $\mathrm{T} 12$ & S1 & $\mathrm{mm}$ \\
\hline L-CVA & Lumbar coronal distance between the reflective markers & $\mathrm{T} 12$ & S1 & $\mathrm{mm}$ \\
\hline S-SVA & Whole spinal sagittal distance between the reflective markers & $\mathrm{C} 7$ & S1 & $\mathrm{mm}$ \\
\hline S-CVA & Whole spinal coronal distance between the reflective markers & $\mathrm{C7}$ & S1 & $\mathrm{mm}$ \\
\hline T-SA & $\begin{array}{l}\text { Thoracic sagittal angle between the vertical axis and the line } \\
\text { connecting the spinal markers }\end{array}$ & C7 & $\mathrm{T} 12$ & $\begin{array}{l}\text { angle } \\
\left({ }^{\circ}\right)\end{array}$ \\
\hline T-CA & $\begin{array}{l}\text { Thoracic coronal angle between the vertical axis and the line } \\
\text { connecting the spinal markers }\end{array}$ & C7 & T12 & $\begin{array}{l}\text { angle } \\
\left({ }^{\circ}\right)\end{array}$ \\
\hline L-SA & $\begin{array}{l}\text { Lumbar sagittal angle between the vertical axis and the line } \\
\text { connecting the spinal markers }\end{array}$ & T12 & S1 & $\begin{array}{l}\text { angle } \\
\left({ }^{\circ}\right)\end{array}$ \\
\hline L-CA & $\begin{array}{l}\text { Lumbar coronal angle between the vertical axis and the line } \\
\text { connecting the spinal markers }\end{array}$ & T12 & S1 & $\begin{array}{l}\text { angle } \\
\left({ }^{\circ}\right)\end{array}$ \\
\hline S-SA & $\begin{array}{l}\text { Whole spinal sagittal angle between the vertical axis and the } \\
\text { line connecting the spinal markers }\end{array}$ & C7 & S1 & $\begin{array}{l}\text { angle } \\
\left({ }^{\circ}\right)\end{array}$ \\
\hline S-CA & $\begin{array}{l}\text { Whole spinal coronal angle between the vertical axis and the } \\
\text { line connecting the spinal markers }\end{array}$ & C7 & S1 & $\begin{array}{l}\text { angle } \\
\left({ }^{\circ}\right)\end{array}$ \\
\hline P-SA & $\begin{array}{l}\text { Sagittal angle between the horizontal axis and the line } \\
\text { connecting the reflective markers on the ASIS and PSIS }\end{array}$ & ASIS & PSIS & $\begin{array}{l}\text { angle } \\
\left({ }^{\circ}\right)\end{array}$ \\
\hline P-CA & $\begin{array}{l}\text { Coronal angle between the horizontal axis and the line } \\
\text { connecting the reflective markers on the ASIS and PSIS }\end{array}$ & ASIS & PSIS & $\begin{array}{l}\text { angle } \\
\left({ }^{\circ}\right)\end{array}$ \\
\hline T-P SA & $\begin{array}{l}\text { Thoracic sagittal angle between the line connecting the spinal } \\
\text { markers and the line connecting the reflective markers on the } \\
\text { ASIS and PSIS }\end{array}$ & $\mathrm{C7}$ & T12 & $\begin{array}{l}\text { angle } \\
\left({ }^{\circ}\right)\end{array}$ \\
\hline T-P CA & $\begin{array}{l}\text { Thoracic coronal angle between the line connecting the spinal } \\
\text { markers and the line connecting the reflective markers on the } \\
\text { ASIS and PSIS }\end{array}$ & C7 & $\mathrm{T} 12$ & $\begin{array}{l}\text { angle } \\
\left({ }^{\circ}\right)\end{array}$ \\
\hline L-P SA & $\begin{array}{l}\text { Lumbar sagittal angle between the line connecting the spinal } \\
\text { markers and the line connecting the reflective markers on the } \\
\text { ASIS and PSIS }\end{array}$ & T12 & S1 & $\begin{array}{l}\text { angle } \\
\left({ }^{\circ}\right)\end{array}$ \\
\hline
\end{tabular}

SVA, sagittal vertical axis; CVA, coronal vertical axis; SA, sagittal angle; CA, coronal angle; P SA, pelvic sagittal angle; P CA, pelvic coronal angle; ASIS, anterior superior iliac spine; PSIS, posterior superior iliac spine 


\begin{tabular}{|lllll|}
\hline Parameter & Definition & Marker & Unit \\
\hline L-P CA & $\begin{array}{l}\text { Lumbar coronal angle between the line connecting the spinal } \\
\text { markers and the line connecting the reflective markers on the } \\
\text { ASIS and PSIS }\end{array}$ & T12 & S1 & $\begin{array}{c}\text { angle } \\
\left(^{\circ}\right)\end{array}$ \\
\hline S-P SA & $\begin{array}{l}\text { Whole spinal sagittal angle between the line connecting the } \\
\text { spinal markers and the line connecting the reflective markers } \\
\text { on the ASIS and PSIS }\end{array}$ & C7 & S1 & $\begin{array}{l}\text { angle } \\
\left(^{\circ}\right)\end{array}$ \\
\hline S-P CA & $\begin{array}{l}\text { Whole spinal coronal angle between the line connecting the } \\
\text { spinal markers and the line connecting the reflective markers } \\
\text { on the ASIS and PSIS }\end{array}$ & C7 & S1 & $\begin{array}{l}\text { angle } \\
\left(^{\circ}\right)\end{array}$ \\
\hline $\begin{array}{l}\text { SVA, sagittal vertical axis; CVA, coronal vertical axis; SA, sagittal angle; CA, coronal angle; P SA, pelvic } \\
\text { sagittal angle; P CA, pelvic coronal angle; ASIS, anterior superior iliac spine; PSIS, posterior superior } \\
\text { iliac spine }\end{array}$ & & \\
\hline
\end{tabular}

Figure 5 shows the relationship between each spinal marker and each dynamic spinal parameter.

These spinal parameters were recorded continuously during all trials. The mean values of the parameters for each lap were calculated as the parameters for the lap. Thoracic kyphosis and pelvic anteversion are considered to progress with walking. Therefore, we sampled the parameters of the final lap, which is considered to exhibit the most exacerbated alignment.

\section{Statistical analysis}

All continuous values are described as mean \pm standard deviation (SD). We compared all parameters between the group with PJK(+) and the group with PJK(-) using an unpaired Student $t$ test. A ShapiroWilk test was used for each dynamic parameter to evaluate the normal distribution. The post hoc analysis for the Student $t$ test was performed using G-power software (version 3.1.9.6, Dusseldorf, Germany). Preoperative variables associated with PJK $(P<0.10)$ on univariate analysis were included in a multivariate logistic regression model with forward stepwise algorithms. Variables that did not fit the model significantly were rejected. Odds ratios (ORs) and $95 \%$ confidence intervals (Cls) were calculated. A Hosmer-Lemeshow test was used as a statistical test for goodness of fit of the logistic regression model. All statistical analyses except for the post hoc power analyses were conducted using JMP statistical software for Windows (version 16; SAS, Cary, NC, USA). $P<0.05$ was considered significant for tests of statistical difference.

\section{Declarations}

\section{Author contributions (names must be given as initials)}

T.A. conceived this study. Kou.M., Mas.K., M.Y. contributed to the study design. T.A., Kou.M., H.K. coordinated the data collection and conducted analysis and data interpretation. T.A. drafted the initial manuscript. Mas.K. T.F., H.T., H.N., Y.S., K.S., F.E., Mam.K., Ken. M. gave critical comments on the draft of the manuscript. All authors read and approved the final version of the manuscript. 


\section{Funding}

This study was supported by AO Spine Asia Pacific 2020 Research National 23 Grant (Grant no: AOSJP(R)2020-08) , 5000 CHF

\section{Additional Information (including a Competing Interests Statement)}

Competing interests: The authors declare no competing interests.

\section{References}

1 Lee, C. H. et al. Effectiveness of deformity-correction surgery for primary degenerative sagittal imbalance: a meta-analysis. J Neurosurg Spine 27, 540-551, doi:10.3171/2017.3.Spine161134 (2017).

2 Glattes, R. C. et al. Proximal Junctional Kyphosis in Adult Spinal Deformity Following Long Instrumented Posterior Spinal Fusion: Incidence, Outcomes, and Risk Factor Analysis. Spine 30, 16431649, doi:10.1097/01.brs.0000169451.76359.49 (2005).

3 Cho, S. K., Shin, J. I. \& Kim, Y. J. Proximal junctional kyphosis following adult spinal deformity surgery. Eur Spine J 23, 2726-2736, doi:10.1007/s00586-014-3531-4 (2014).

$4 \quad$ Han, S. et al. Rod stiffness as a risk factor of proximal junctional kyphosis after adult spinal deformity surgery: comparative study between cobalt chrome multiple-rod constructs and titanium alloy two-rod constructs. The Spine Journal 17, 962-968, doi:10.1016/j.spinee.2017.02.005 (2017).

5 Kim, J. S. et al. Surgical, Radiographic, and Patient-Related Risk Factors for Proximal Junctional Kyphosis: A Meta-Analysis. Global Spine J 9, 32-40, doi:10.1177/2192568218761362 (2019).

6 Lau, D. et al. Proximal junctional kyphosis and failure after spinal deformity surgery: a systematic review of the literature as a background to classification development. Spine (Phila Pa 1976) 39, 20932102, doi:10.1097/brs.0000000000000627 (2014).

7 Liu, F. Y. et al. Incidence and risk factors for proximal junctional kyphosis: a meta-analysis. Eur Spine J 25, 2376-2383, doi:10.1007/s00586-016-4534-0 (2016).

8 Lafage, R. et al. Risk Factor Analysis for Proximal Junctional Kyphosis After Adult Spinal Deformity Surgery: A New Simple Scoring System to Identify High-Risk Patients. Global Spine J 10, 863870, doi:10.1177/2192568219882350 (2020).

9 Luo, M. et al. Upper Thoracic versus Lower Thoracic as Site of Upper Instrumented Vertebrae for Long Fusion Surgery in Adult Spinal Deformity: A Meta-Analysis of Proximal Junctional Kyphosis. World Neurosurg 102, 200-208, doi:10.1016/j.wneu.2017.02.126 (2017). 
10 Chen, S., Luo, M., Wang, Y. \& Liu, H. Stopping at Sacrum Versus Nonsacral Vertebra in Long Fusion Surgery for Adult Spinal Deformity: Meta-Analysis of Revision with Minimum 2-Year Follow-Up. World Neurosurg, doi:10.1016/j.wneu.2018.12.102 (2018).

11 Raman, T., Miller, E., Martin, C. T. \& Kebaish, K. M. The effect of prophylactic vertebroplasty on the incidence of proximal junctional kyphosis and proximal junctional failure following posterior spinal fusion in adult spinal deformity: a 5-year follow-up study. Spine J 17, 1489-1498, doi:10.1016/j.spinee.2017.05.017 (2017).

12 Park, S.-J., Lee, C.-S., Park, J.-S. \& Lee, K.-J. Should Thoracolumbar Junction Be Always Avoided as Upper Instrumented Vertebra in Long Instrumented Fusion for Adult Spinal Deformity?: Risk Factor Analysis for Proximal Junctional Failure. Spine 45, 686-693, doi:10.1097/brs.0000000000003364 (2020).

13 Arima, H. et al. Discrepancy Between Standing Posture and Sagittal Balance During Walking in Adult Spinal Deformity Patients. Spine (Phila Pa 1976) 42, E25-e30, doi:10.1097/brs.0000000000001709 (2017).

14 Bae, J., Theologis, A. A., Jang, J. S., Lee, S. H. \& Deviren, V. Impact of Fatigue on Maintenance of Upright Posture: Dynamic Assessment of Sagittal Spinal Deformity Parameters After Walking 10 Minutes. Spine (Phila Pa 1976) 42, 733-739, doi:10.1097/brs.0000000000001898 (2017).

15 Haddas, R., Hu, X. \& Lieberman, I. H. The Correlation of Spinopelvic Parameters With Biomechanical Parameters Measured by Gait and Balance Analyses in Patients With Adult Degenerative Scoliosis. Clin Spine Surg 33, E33-E39, doi:10.1097/bsd.0000000000000939 (2020).

16 Miura, K. et al. Successful detection of postoperative improvement of dynamic sagittal balance with a newly developed three-dimensional gait motion analysis system in a patient with iatrogenic flatback syndrome: A case report. J Clin Neurosci 53, 241-243, doi:10.1016/j.jocn.2018.04.051 (2018).

17 Miura, K. et al. Thoracic kyphosis and pelvic anteversion in patients with adult spinal deformity increase while walking: analyses of dynamic alignment change using a three-dimensional gait motion analysis system. Eur Spine J 29, 840-848, doi:10.1007/s00586-020-06312-y (2020).

18 Hyun, S.-J., Kim, Y. J. \& Rhim, S.-C. Patients with proximal junctional kyphosis after stopping at thoracolumbar junction have lower muscularity, fatty degeneration at the thoracolumbar area. The Spine Journal 16, 1095-1101, doi:10.1016/j.spinee.2016.05.008 (2016).

19 Yuan, L. et al. Risk Factors Associated With Failure to Reach Minimal Clinically Important Difference After Correction Surgery in Patients With Degenerative Lumbar Scoliosis. Spine (Phila Pa 1976) 45, E1669-e1676, doi:10.1097/brs.0000000000003713 (2020).

20 Sakuma, T. et al. Incidence, Risk Factors, and Prevention Strategy for Proximal Junctional Kyphosis in Adult Spinal Deformity Surgery. Spine Surg Relat Res 5, 75-80, doi:10.22603/ssrr.2020-0093 
(2021).

21 Lafage, R. et al. Defining Spino-Pelvic Alignment Thresholds: Should Operative Goals in Adult Spinal Deformity Surgery Account for Age? Spine (Phila Pa 1976) 41, 62-68, doi:10.1097/BRS.0000000000001171 (2016).

22 Yamato, Y. et al. Rigorous Correction of Sagittal Vertical Axis Is Correlated With Better ODI Outcomes After Extensive Corrective Fusion in Elderly or Extremely Elderly Patients With Spinal Deformity. Spine deformity 7, 610-618, doi:10.1016/j.jspd.2018.11.001 (2019).

23 Ham, D. W. et al. Risk factors for acute proximal junctional kyphosis after adult spinal deformity surgery in preoperative motion analysis. Eur Spine J, doi:10.1007/s00586-021-06830-3 (2021).

24 Schmid, S. et al. Using Skin Markers for Spinal Curvature Quantification in Main Thoracic Adolescent Idiopathic Scoliosis: An Explorative Radiographic Study. PloS one 10, e0135689, doi:10.1371/journal.pone.0135689 (2015).

25 Schwab, F., Patel, A., Ungar, B., Farcy, J.-P. \& Lafage, V. Adult Spinal Deformity-Postoperative Standing Imbalance: How Much Can You Tolerate? An Overview of Key Parameters in Assessing Alignment and Planning Corrective Surgery. Spine 35, 2224-2231, doi:10.1097/BRS.0b013e3181 ee6bd4 (2010).

26 Yagi, M., King, A. B. \& Boachie-Adjei, O. Characterization of osteopenia/osteoporosis in adult scoliosis: does bone density affect surgical outcome? Spine (Phila Pa 1976) 36, 1652-1657, doi:10.1097/BRS.0b013e31820110b4 (2011).

27 Lochmüller, E. M. et al. Mechanical strength of the thoracolumbar spine in the elderly: prediction from in situ dual-energy X-ray absorptiometry, quantitative computed tomography (QCT), upper and lower limb peripheral QCT, and quantitative ultrasound. Bone 31, 77-84, doi:10.1016/s8756-3282(02)00792-5 (2002).

28 Schwab, F. et al. The comprehensive anatomical spinal osteotomy classification. Neurosurgery 74, 112-120; discussion 120, doi:10.1227/NEU.0000000000000182o (2014).

\section{Figures}


36 patients underwent ASD surgery

and preoperative gait analysis in $2015-2020$

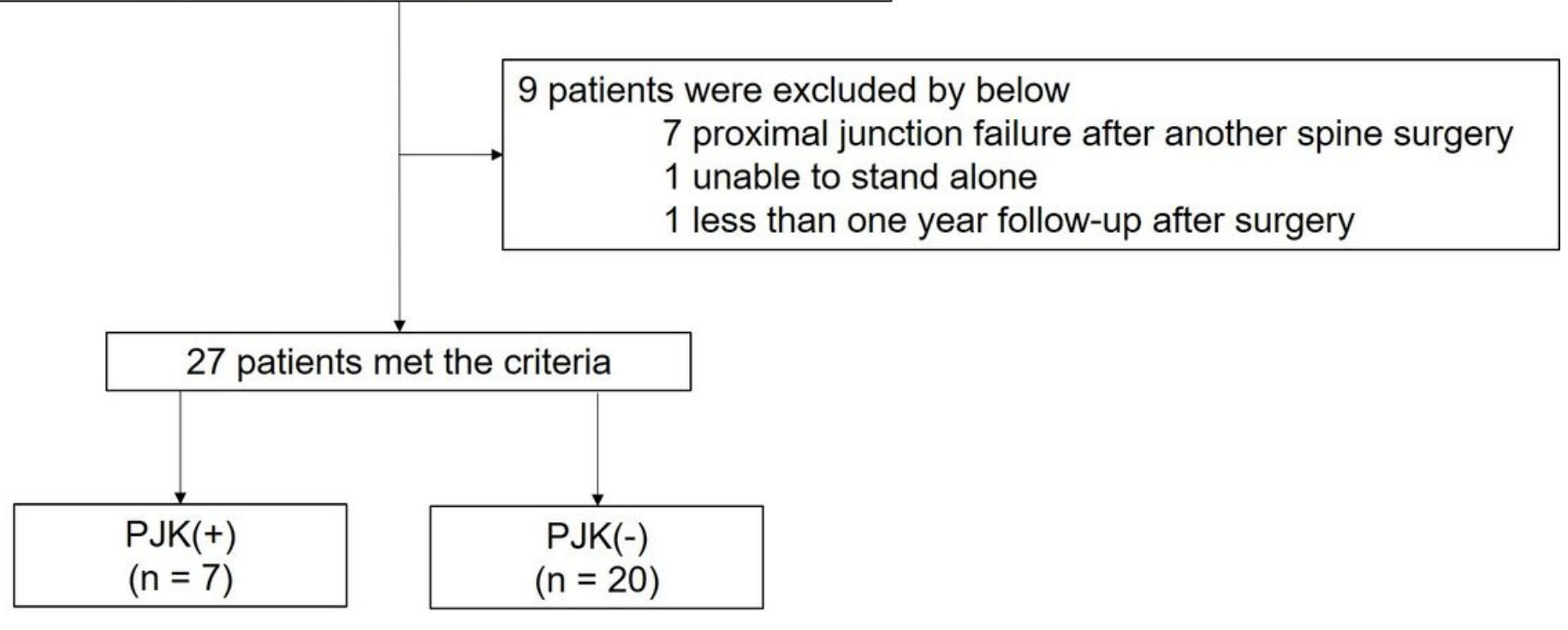

\section{Figure 1}

Patient inclusion flow chart PJK $(+)$ is the patient group with proximal junctional angle (PJA) $>10^{\circ}$ soon after surgery and $>10^{\circ}$ progression of PJA. PJK( $(-)$ is the patient group with proximal junctional angle (PJA) $<10^{\circ}$ soon after surgery or $<10^{\circ}$ progression of PJA



Figure 2 
Representative case of PJK Preoperative standing X-ray images indicated the following spinal

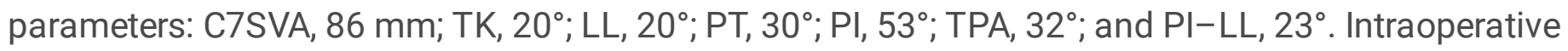
image showing that PJA was 20.2. PJA developed in postoperative month 6. Finally, PJA was $31.2^{\circ}$ at the first year postoperatively and the change was $11.2^{\circ}$. This patient complained of implant eminence and pain in the proximal junctional area, but refused reoperation.

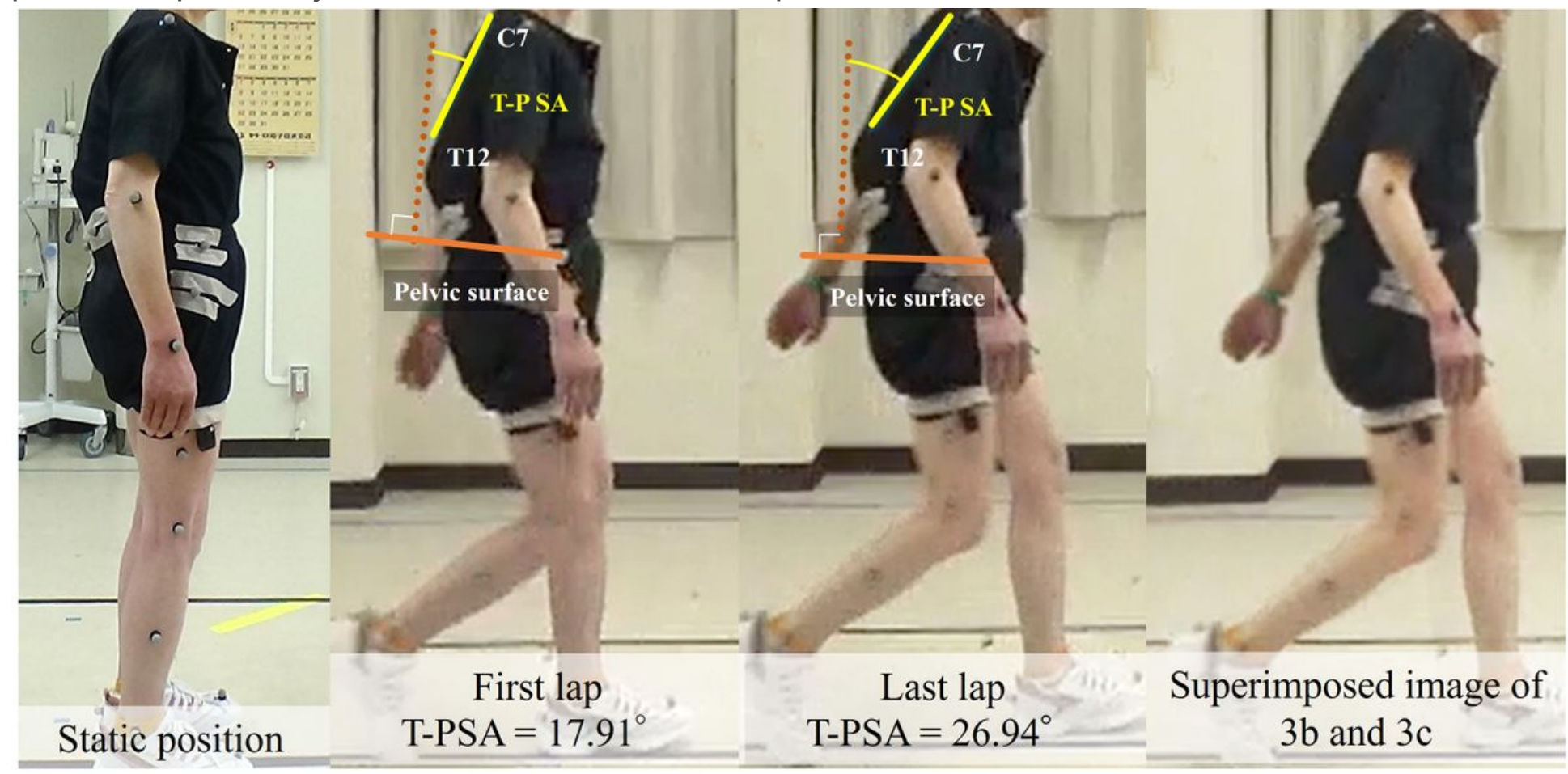

Fig. 3a

Fig. $3 b$

Fig. 3c

Fig. 3d

\section{Figure 3}

Gait analysis of representative case This figure indicates the posture during gait analysis. Before starting to walk, the patient seemed relatively balanced in an upright standing position before gait analysis (Fig 3a). When starting the walk, the thoracic part began to lean forward in the first lap (Fig 3b). In the final lap soon before quitting the trial, the tilt of the thoracic spine leaned further forward (Fig. 3c). A superimposed image of the posture during gait shows the change of the thoracic spine tilt between the first (T-P SA $17.91^{\circ}$ ) and the final lap (T-P SA 26.94) (Fig. 3d). 


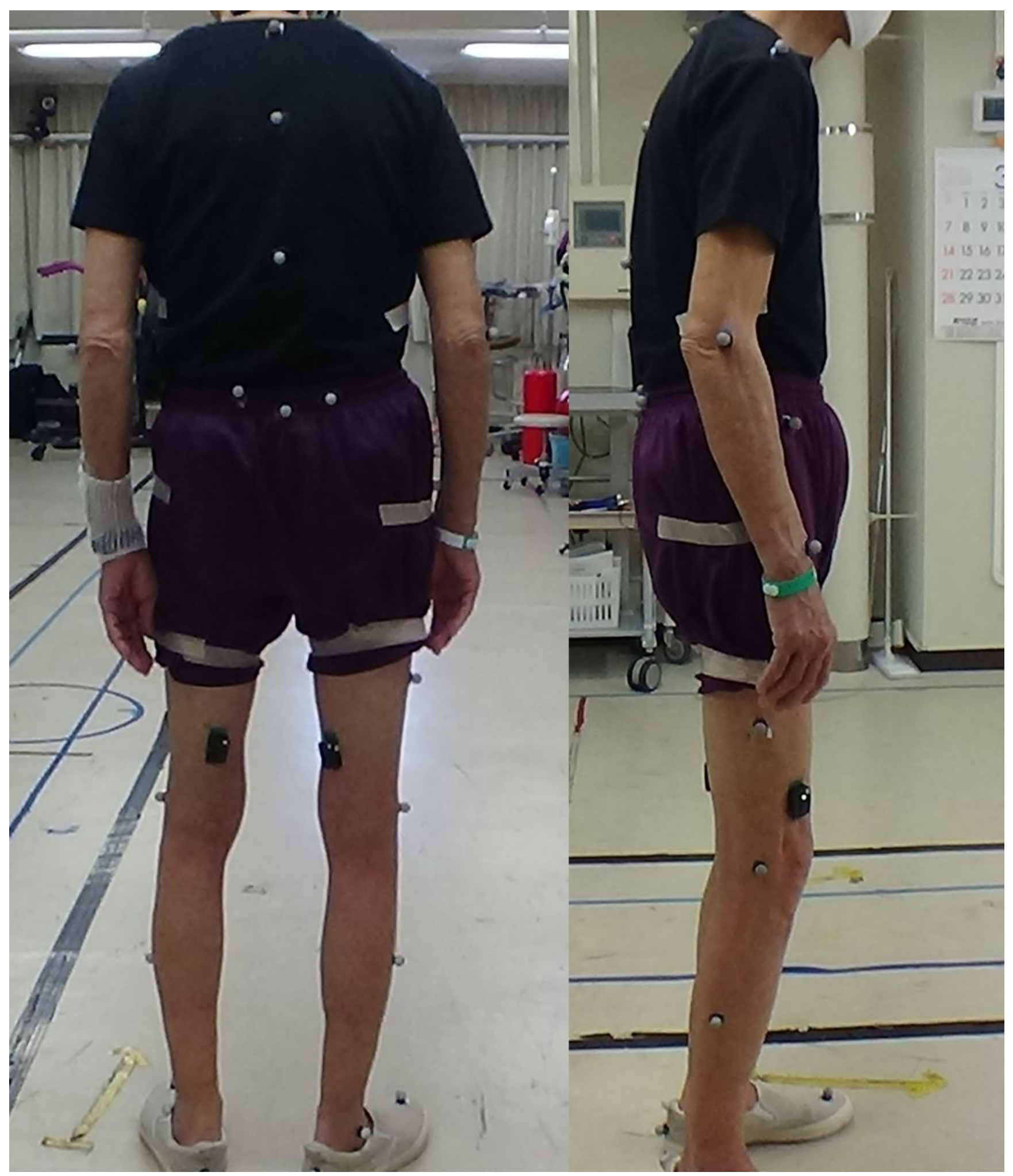

Figure 4

Reflective markers were placed on the spinal spinous and pelvic processes. 



Figure 5

a. Dynamic sagittal spinal parameters The dotted white line indicates a perpendicular line to the floor. The dotted brown line indicates the perpendicular line to the surface created from the 2 ASIS and 2 PSIS points (pelvic surface). SVA was defined by the sagittal distance between C7-T12 (T-SVA), T12-S1 (LSVA) and C7-S1(S-SVA). SA was defined by the sagittal angle of C7-T12 line (T-SA), T12-S1 line (LSA), and C7-S1 line (S-SA) from the perpendicular line. PSA was defined by the sagittal angle between the floor and the pelvic surface. T-PSA, L-PSA and S-PSA were defined by the sagittal angle of C7-T12 line (T-P SA), T12-S1 line (L-P SA), and C7-S1 line (S-P SA) from the perpendicular line to the pelvic surface. T, thoracic; L, lumbar; S, whole spinal; SVA, sagittal vertical axis; SA, sagittal angle; P SA, pelvic sagittal angle; ASIS, anterior superior iliac spine; PSIS, posterior superior iliac spine b. Dynamic coronal 
spinal parameters The dotted white line indicates a perpendicular line to the floor. The dotted brown line indicates the perpendicular line to the surface created from the 2 ASIS and 2 PSIS points (pelvic surface). CVA was defined by the coronal distance between C7-T12 (T-CVA), T12-S1 (L-CVA), and C7-S1 (S-CVA). CA was defined by the coronal angle of C7-T12 line (T-CA), T12-S1 line (L-CA), and C7-S1 line (S-CA) from the perpendicular line. PCA was defined by the coronal angle between the floor and the pelvic surface. T-PCA, L-PCA, and S-PCA were defined by the sagittal angle of C7-T12 line (T-P SA), T12-S1 line (L-P SA), and C7-S1 line (S-P SA) from the perpendicular line to the pelvic surface. T, thoracic; $L$, lumbar; $\mathrm{S}$, whole spinal; CVA, coronal vertical axis; CA, coronal angle; P CA, pelvic coronal angle; ASIS, anterior superior iliac spine; PSIS, posterior superior iliac spine. 